\title{
A biospectroscopic assignment technique for gum cancer cell membrane DNA/RNA reconstituted in magnetically aligned gum cancer cells for solid-state NMR analysis of gum cancer cell membrane DNA/RNA and nucleic acids aggregates by proton detected spectroscopy
}

\author{
Alireza Heidari ${ }^{1,2 *}$ and Ricardo Gobato ${ }^{3}$ \\ ${ }^{1}$ Faculty of Chemistry, California South University, 14731 Comet St. Irvine, CA 92604, USA \\ ${ }^{2}$ American International Standards Institute, Irvine, CA 3800, USA \\ ${ }^{3}$ Green Land Landscaping and Gardening, Seedling Growth Laboratory, 86130-000, Parana, Brazil
}

\begin{abstract}
In the current research, a biospectroscopic assignment technique for gum cancer cell membrane DNA/RNA reconstituted in magnetically aligned gum cancer cells for solid-state NMR analysis of gum cancer cell membrane DNA/RNA and nucleic acids aggregates by proton detected spectroscopy are considered. In recent years, biospectroscopic assignment technique for gum cancer cell membrane DNA/RNA reconstituted in magnetically aligned gum cancer cells for solid-state NMR analysis of gum cancer cell membrane DNA/RNA and nucleic acids aggregates by proton detected spectroscopy has gained widespread acceptance in applications that span from the rapid identification of unknown components to detailed characterization of materials and biological samples. The technique's breadth of application is too wide to reference here, but examples include quality control (QC) testing and verification of high purity chemicals and raw materials in the pharmaceuticals and food industries, investigation of counterfeit drugs, classification of polymers and plastics, characterization of tumors, and the detection of molecular biomarkers in disease diagnostics and theranostics.
\end{abstract}

\section{Introduction}

Evidence for balanced cell growth where protein and DNA/RNA content rise at a constant relative rate with respect to DNA/RNA content with increasing grade of gum cancer and with malignant transformation, is derived from analysis of biospectroscopic assignment technique for gum cancer cell membrane DNA/RNA reconstituted in magnetically aligned gum cancer cells for solid-state NMR analysis of gum cancer cell membrane DNA/RNA and nucleic acids aggregates by proton detected of malignant lymphoma and transformed fibroblasts within the literature. This evidence is consistent with flow cytometry derived DNA/ RNA-protein relationships, and supports the biosynthetic connection proposed here between the DNA/RNA ratio. The field of gum cancer diagnostics has always been one of the most complex and challenging areas in biomedical research, and there has been an increasing demand for more advanced clinical diagnostic equipment over the past four decades. In this thesis, a custom-made coaxial bioimpedance sensor was used, in combination with computer-aided pattern recognition tools, to identify gum cancer in tissue samples obtained from the formalin-fixed kidney of a 60 year-old male patient. Impedance data was collected using the coaxial sensor at 401 logarithmically-spaced frequency points ranging from $10 \mathrm{kHz}$ to $100 \mathrm{MHz}$. biospectroscopic assignment technique for gum cancer cell membrane DNA/RNA reconstituted in magnetically aligned gum cancer cells for solid-state NMR analysis of gum cancer cell membrane DNA/RNA and nucleic acids aggregates by proton detected were employed to test whether bioimpedance could discriminate between the cancer and non-cancer tissue within the specimen. The classification model was trained using measurement data from cancer and non-cancer sections of the sample. The models were then tested using cross validation techniques. It was concluded that the Naive Bayes classifier could discriminate gum cancer from normal samples using capacitance and conductance data [1-10].

\section{Results and discussion}

Biospectroscopic assignment technique for gum cancer cell membrane DNA/RNA reconstituted in magnetically aligned gum cancer cells for solid-state NMR analysis of gum cancer cell membrane DNA/RNA and nucleic acids aggregates by proton detected are proving to be invaluable tools in the field of biomedical research and clinical diagnostics. The robust, compact, fit-for-purpose biospectroscopic assignment technique for gum cancer cell membrane DNA/RNA

*Correspondence to: Alireza Heidari, Faculty of Chemistry, California South University, 14731 Comet St. Irvine, CA 92604; American International Standards Institute, Irvine, CA 3800, USA, E-mail: Scholar.Researcher.Scientist@gmail. com; Alireza.Heidari@calsu.us; Central@aisi-usa.org

Key words: biospectroscopy, assignment technique, gum cancer cell membrane, DNA/RNA, solid-state NMR analysis, nucleic acids, proton detected spectroscopy

Received: February 15, 2021; Accepted: March 08, 2021; Published: March 13, 2021 
Heidari A (2021) A biospectroscopic assignment technique for gum cancer cell membrane DNA/RNA reconstituted in magnetically aligned gum cancer cells for solid-state NMR analysis of gum cancer cell membrane DNA/RNA and nucleic acids aggregates by proton detected spectroscopy

reconstituted in magnetically aligned gum cancer cells for solid-state NMR analysis of gum cancer cell membrane DNA/RNA and nucleic acids aggregates by proton detected are appropriate for use in surgical procedures to help surgeons assess tumors and allow rapid decisions to be made. Biospectroscopic assignment technique for gum cancer cell membrane DNA/RNA reconstituted in magnetically aligned gum cancer cells for solid-state NMR analysis of gum cancer cell membrane DNA/RNA and nucleic acids aggregates by proton detected are also being developed for molecular diagnostic testing to detect and measure human gum cancer biomarkers. Based on the biospectroscopic assignment technique for gum cancer cell membrane DNA/RNA reconstituted in magnetically aligned gum cancer cells for solid-state NMR analysis of gum cancer cell membrane DNA/RNA and nucleic acids aggregates by proton detected, this could potentially change the way bioassays are performed to improve both the sensitivity and reliability of testing. The two applications highlighted in this review together with other examples of the use of biospectroscopic assignment technique for gum cancer cell membrane DNA/RNA reconstituted in magnetically aligned gum cancer cells for solid-state NMR analysis of gum cancer cell membrane DNA/RNA and nucleic acids aggregates by proton detected in biomedical research areas, such as the identification of bacterial infections, are clearly going to make the technique an important part of the medical toolbox, as we continually strive to improve diagnostic techniques and bring a better health care system to patients (Figure 1).

\section{Conclusion}

Biospectroscopic assignment technique for gum cancer cell membrane DNA/RNA reconstituted in magnetically aligned gum cancer cells for solid-state NMR analysis of gum cancer cell membrane DNA/RNA and nucleic acids aggregates by proton detected is a more sensitive version of biospectroscopic assignment technique for gum cancer cell membrane DNA/RNA reconstituted in magnetically aligned gum cancer cells for solid-state NMR analysis of gum cancer cell membrane DNA/RNA and nucleic acids aggregates by proton detected, relying on the principle that biospectroscopic assignment technique for gum cancer cell membrane DNA/RNA reconstituted in magnetically aligned gum cancer cells for solid-state NMR analysis of gum cancer cell membrane DNA/RNA and nucleic acids aggregates by proton detected is enhanced by several orders of magnitude when the sample is deposited onto a roughened metallic surface. The benefit of

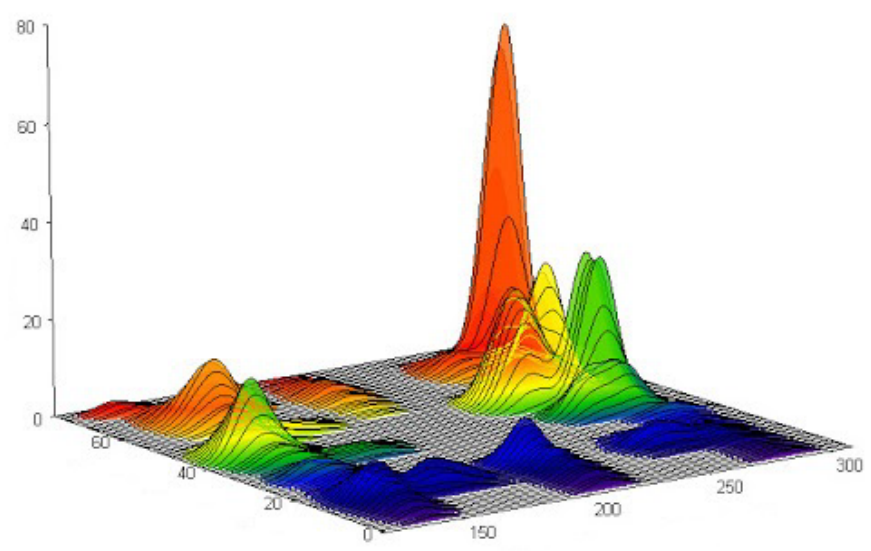

Figure 1. Gum cancer cell membrane DNA/RNA reconstituted in magnetically aligned gum cancer cells for solid-state NMR analysis of gum cancer cell membrane DNA/RNA and nucleic acids aggregates by proton detected spectroscopy biospectroscopic assignment technique for gum cancer cell membrane DNA/RNA reconstituted in magnetically aligned gum cancer cells for solid-state NMR analysis of gum cancer cell membrane DNA/ RNA and nucleic acids aggregates by proton detected for these types of applications is that it provides well-defined, distinct spectral information, enabling characterization of various states of a disease to be detected at much lower levels. Some of the many applications of biospectroscopic assignment technique for gum cancer cell membrane DNA/RNA reconstituted in magnetically aligned gum cancer cells for solid-state NMR analysis of gum cancer cell membrane DNA/RNA and nucleic acids aggregates by proton detected for biomedical monitoring include.

\section{Acknowledgments}

This study was supported by the Cancer Research Institute (CRI) Project of Scientific Instrument and Equipment Development, the National Natural Science Foundation of the United Sates, the International Joint BioSpectroscopy Core Research Laboratory Program supported by the California South University (CSU), and the Key project supported by the American International Standards Institute (AISI), Irvine, California, USA.

\section{References}

1. A. Heidari, C. Brown, "Study of Composition and Morphology of Cadmium Oxide (CdO) Nanoparticles for Eliminating Cancer Cells", J Nanomed Res., Volume 2, Issue 5, 20 Pages, 2015.

2. A. Heidari, C. Brown, "Study of Surface Morphological, Phytochemical and Structural Characteristics of Rhodium (III) Oxide (Rh, $\mathrm{O}_{3}$ ) Nanoparticles", International Journal of Pharmacology, Phytochemistry and Ethnomedicine, Volume 1, Issue 1, Pages 15-19, 2015.

3. A. Heidari, “An Experimental Biospectroscopic Study on Seminal Plasma in Determination of Semen Quality for Evaluation of Male Infertility”, Int J Adv Technol 7: e007, 2016.

4. A. Heidari, "Extraction and Preconcentration of N-Tolyl-Sulfonyl-PhosphoramidSaeure-Dichlorid as an Anti-Cancer Drug from Plants: A Pharmacognosy Study", J Pharmacogn Nat Prod 2: e103, 2016.

5. A. Heidari, "A Thermodynamic Study on Hydration and Dehydration of DNA and RNA-Amphiphile Complexes”, J Bioeng Biomed Sci S: 006, 2016.

6. A. Heidari, "Computational Studies on Molecular Structures and Carbonyl and Ketene Groups' Effects of Singlet and Triplet Energies of Azidoketene $O=C=C H-N N N$ and Isocyanatoketene $O=C=C H-N=C=O$ ", J Appl Computat Math 5: e142, 2016.

7. A. Heidari, "Study of Irradiations to Enhance the Induces the Dissociation of Hydrogen Bonds between Peptide Chains and Transition from Helix Structure to Random Coil Structure Using ATR-FTIR, Raman and 'HNMR Spectroscopies", J Biomol Res Ther 5: e146, 2016

8. A. Heidari, "Future Prospects of Point Fluorescence Spectroscopy, Fluorescence Imaging and Fluorescence Endoscopy in Photodynamic Therapy (PDT) for Cancer Cells", J Bioanal Biomed 8: e135, 2016.

9. A. Heidari, "A Bio-Spectroscopic Study of DNA Density and Color Role as Determining Factor for Absorbed Irradiation in Cancer Cells", Adv Cancer Prev 1: e102, 2016.

10. A. Heidari, "Manufacturing Process of Solar Cells Using Cadmium Oxide (CdO) and Rhodium (III) Oxide $\left(\mathrm{Rh}_{2} \mathrm{O}_{3}\right)$ Nanoparticles", J Biotechnol Biomater 6: e125, 2016.:2, 2016.

Copyright: (C2021 Heidari A. This is an open-access article distributed under the terms of the Creative Commons Attribution License, which permits unrestricted use, distribution, and reproduction in any medium, provided the original author and source are credited. 